\title{
Copulas for Statistical Signal Processing (Part I): Extensions and Generalization
}

\author{
Xuexing Zeng ${ }^{1}$, Jinchang Ren $^{1}{ }^{*}$, Zheng Wang ${ }^{2}$, Stephen Marshall ${ }^{1}$, Tariq Durrani ${ }^{1}$ \\ ${ }^{1}$ Centre for excellence in Signal and Image Processing (CeSIP), \\ Dept. of Electronic and Electrical Engineering, \\ University of Strathclyde, Glasgow, United Kingdom \\ ${ }^{2}$ School of Computer Software, Tianjin University, Tianjin, 300072, China
}

\begin{abstract}
Existing works on multivariate distributions mainly focus on limited distribution functions and require that the associated marginal distributions belong to the same family. Although this simplifies problems, it may fail to deal with practical cases when the marginal distributions are arbitrary. To this end, copula function is employed since it provides a flexible way in decoupling the marginal distributions and dependence structure for random variables. Among different copula functions, most researches focus on Gaussian, Student's $t$ and Archimedean copulas for simplicity. In this paper, to extend bivariate copula families, we have constructed new bivariate copulas for exponential, Weibull and Rician distributions. We have proved that the three copula functions of exponential, Rayleigh and Weibull distributions are equivalent, constrained by only one parameter, thus greatly facilitating practical applications of them. We have also proved that the copula function of log-normal distribution is equivalent to the Gaussian copula. Moreover, we have derived the Rician copula with two parameters. In addition, the modified Bessel function or incomplete Gamma function with double integrals in the copula functions are simplified by single integral or infinite series for computational efficiency. Associated copula density functions for exponential, Rayleigh, Weibull, log-normal, Nakagami-m and Rician distributions are also derived.
\end{abstract}

Index Terms - Copulas; statistical signal processing; parameter estimation; arbitrary marginal distributions

*Corresponding Author:

Dr. J. Ren,

Centre for excellence in Signal and Image Processing

Dept. of Electronic and Electrical Engineering\#

University of Strathclyde,

204 George Street, Glasgow,

United Kingdom

Email: Jinchang.Ren@strath.ac.uk

Phone: +44-141-5482384 


\section{INTRODUCTION}

In general, the marginal distribution functions can be derived through straightforward integration from a known multivariate joint distribution function. However, conversely, a unique joint distribution is not readily available from the marginal distributions. Although many multivariate distribution functions have been defined, in most cases the marginal distributions are assumed to be within the same family such as multivariate Gaussian distribution. In practical situations, this assumption does not always hold true, as practically the marginal distributions can be arbitrary and may belong to different families of probability distributions. For example, the probability distributions of pixel intensities for infrared image and visible images of the same objective are usually different. Consequently, some popular multivariate distributions such as the multivariate Gaussian distribution may not be the ideal choice in modeling real problems since it can only capture the linear dependence between random variables.

Copula functions, however, make it possible to capture more sophisticated non-linear dependencies between random variable [1], [2]. Copulas have provided great potential in statistics and probability theory and have been successfully applied in many applications covering finance [3], signal processing [4]-[17], and communications [18]-[19]. Let $F_{X Y}(x, y)$ be a joint cumulative distribution function (cdf) with two marginal cdfs $F_{X}(x)$ and $F_{Y}(y)$ of two random variables $x$ and $y$, respectively. According to the Sklar's theorem [1], there exists a copula function $C($.) for any $x$ and $y$ such that

$$
F_{X Y}(x, y)=C\left(F_{X}(x), F_{Y}(y)\right)
$$

If $F_{X}(x)$ and $F_{Y}(y)$ are continuous, the copula function $C($.$) is unique [1, eq. 2.3.1]. A bivariate copula is a bivariate$ cumulative distribution function defined on the unit cube with two uniform marginal distributions on the interval $[0,1]$. Let $u=F_{X}(x)$ and $v=F_{Y}(y)$, the copula function $C(u, v)$ is defined as [1]:

$$
C(u, v)=F_{X Y}(x, y)=F_{X Y}\left[F_{X}^{-1}(u), F_{Y}^{-1}(v)\right]
$$

Note that $F_{X}()$ and $F_{Y}()$ are increasing but may not be strictly increasing, $F_{X}^{-1}()$ and $F_{Y}^{-1}()$ are quasi-inverse functions of $F_{X}()$ and $F_{Y}()$, respectively. If $F_{X}()$ and $F_{Y}()$ are strictly increasing, the case certainly becomes the ordinary inverse. Detailed definition of Quasi-inverse function is given in [1, Sec. 2.3]. Due to the non-uniqueness of the definition of the associated inverse of the cdf for the case of discrete-valued random variables, the corresponding copula also becomes non-unique. As a result, in the remainder of this paper only continuous valued random variables are focused.

A bivariate copula has the following properties [1, Sec. (2.2)]: 
1) For any $u, v \in[0,1], C(u, 0)=C(0, v)=0, C(u, 1)=u$ and $C(1, v)=v$

2) For any $u_{1}, u_{2}, v_{1}, v_{2} \in[0,1]$, and $u_{1} \leq u_{2}, v_{1} \leq v_{2}, C($.$) satisfy C\left(u_{2}, v_{2}\right)-C\left(u_{2}, v_{1}\right) \geq C\left(u_{1}, v_{2}\right)-C\left(u_{1}, v_{1}\right)$

Eq. (3) means that the joint probability is zero if any marginal probability is 0 , and if one marginal probability is 1 , the joint probability is the same as the probability of another marginal probability. Eq. (4) is called 2-increasing property of copula functions [1, Sec. 2.9].

A significant advantage of the copula is that copula functions represent dependence structure without using marginal distributions. Actually, its variables $u$ and $v$ are both uniformly distributed, thus it is straight forward to transform $u$ and $v$ into the marginal variables $x$ and $y$ by the inverse marginal cdf, respectively [1, Sec. 2.9]. This characteristic implies that the marginal distributions can be arbitrary when a suitable dependence structure (copula) is given. Moreover, let $f_{X Y}(x, y)$ represent a joint probability density function (pdf) for random variables $x$ and $y$, and $c(u, v)$ represent copula density function, according to the definition of copula density function, the joint pdf can be obtained as [3, Sec. 4.5]

$$
c(u, v)=\frac{\partial^{2} C(u, v)}{\partial u \partial v}=\frac{\partial^{2} C\left[F_{X}(x), F_{Y}(y)\right]}{\partial F_{X}(x) \partial F_{Y}(y)}=\frac{f_{X Y}(x, y)}{f_{X}(x) f_{Y}(y)}
$$

where $f_{X}(x)$ and $f_{Y}(y)$ represent the marginal pdfs of $x$ and $y$, respectively. This means that the joint pdf can be expressed as the product of a copula density function and arbitrary marginal pdfs if a suitable copula is adopted.

The copulas that are most frequently applied are Gaussian copula [1, eq. 2.3.6], Student's $t$ copula [3, Sec. 4.8.2] and four Archimedean copula [1, Ch4] including Clayton [3, Sec. 4.8.4], Frank [3, Sec. 4.8.4], Gumbel [2, Sec. 4.8.4] and Ali-Mikhail-Haq copulas [1, Sec. 3.3.2]. In [8], [9] and [10], Gaussian copula is considered for supervised classification of synthetic aperture radar (SAR) images, validating biometric authentication and modeling stochastic dependence in large-scale integration of wind power respectively. Gaussian and Student's t copulas are used in [11] and [12] for unsupervised classification of radar signals and fusion of correlated sensor decisions. In [13], four Archimedean copulas, Clayton, Frank, Gumbel and Ali-Mikhali-Haq, are applied for change detection in remote sensing applications. Clayton, Frank, Gumbel, Gaussian and Student's $t$ copulas are used [14] and [15] for location estimation of random signal source and hypothesis testing using heterogeneous data, respectively. In [16], the four Archimedean copulas along with Gaussian and Student's $t$ copulas are employed for supervised SAR image classification. In [17], Rayleigh copula is used for change detection from SAR images.

Note that a copula function represents a specific dependence structure, thus choosing a suitable copula is always crucial for practical applications. Obviously, existing copulas used above are inappropriate to deal with real and complex cases, such as exponential, Rayleigh, Weibull, Nakagami-m and Rician distributions. As a result, there is a strong need to explore new copula 
families, which has motivated our work in this paper to derive and construct new copulas from existing bivariate distributions including exponential, Rayleigh, Weibull, log-normal, Nakagami-m and Rician as these have been widely used in modeling signal processing, communication and other stochastic problems [20]-[25]. Note that copulas of Rayleigh, log-normal and Nakagami-m distributions have been discussed in [4]-[6]. The Rayleigh copula function has been firstly presented with 3 parameters [4], and the number of parameter was then reduced to 1 [5], where its applications can be found in [17] for change detection in SAR images. In this paper, a new form of Rayleigh copula defined by a single parameter is derived. The log-normal copula was firstly given in [5], where in this paper, the log-normal copula is proved to be equivalent to Gaussian copula [1, eq. (2.3.6)]. The detail status about the current studies on bivariate copulas is summarized in Table 1.

As can be seen, there are many N/A cells in the table which indicates new areas to be explored. Basically, we will focus on these unexplored parts, which inevitably form the novelty and main contributions of the paper as highlighted below. Firstly, new copulas families are proposed for exponential, Weibull and Rician distributions as well as a new expression for Rayleigh copula function. Secondly, the relationships among these copulas are revealed to discover the equivalence of log-normal to Gaussian and exponential to Rayleigh and Weibull. Thirdly, the copula density functions for all the six copula functions discussed above are derived.

\section{BIVARIATE COPULA FUNCTIONS}

Several methods for constructing a copula function have been summarized in [2, Sec. 1.14]. Here, we will adopt the inversion method to derive some new copula functions [2, Sec. 1.14.5].

\subsection{Bivariate Gaussian Copula}

The Gaussian copula is perhaps the most popular copula in applications [2]. The bivariate Gaussian copula is defined by two standard Gaussian distributions, and written as: [1, Sec. 2.3], [3, Sec. 4.8.1]:

$$
C(u, v)=\Psi\left(\Phi^{-1}(u), \Phi^{-1}(v)\right)=\frac{1}{2 \pi \sqrt{1-\rho^{2}}} \int_{-\infty}^{\Phi^{-1}(u)} \int_{-\infty}^{\Phi^{-1}(v)} \exp \left[-\frac{s^{2}-2 \rho s t+t^{2}}{2\left(1-\rho^{2}\right)}\right] d s d t
$$

where $\rho \in[-1,1]$ is the only Gaussian copula parameter, $\Phi($.$) denotes the cdf of standard univariate Gaussian distribution and$ $\Psi$ (.) denotes the standard bivariate Gaussian distribution function. The standard Gaussian cdf can be written as $\Phi(z)=\frac{1}{\sqrt{2 \pi}} \int_{-\infty}^{z} \exp \left(\frac{-t^{2}}{2}\right) d t$ and $\Phi^{-1}(u)=\sqrt{2} \operatorname{erf}^{-1}(2 u-1)$. Here erf(.) function is called error function, which is defined as $\operatorname{erf}(x)=\frac{2}{\sqrt{\pi}} \int_{0}^{x} \exp \left(-t^{2}\right) d t$

Actually, according the theorem 2.4.3 in [1], we can prove that the Gaussian copula can be defined by two generalized (non-standard) Gaussian distributions as follows. The theorem 2.4.3 in [1] can be described as: Let $X$ and $Y$ be continuous random 
variables with copula $C_{X Y}$. If $\alpha_{X}$ and $\beta_{Y}$ are strictly increasing on $\operatorname{Ran} \boldsymbol{X}$ and $\boldsymbol{R} a \boldsymbol{Y} Y$, respectively, then $C_{\alpha_{X} \beta_{Y}}=C_{X Y}$. Thus $C_{X Y}$ is invariant under strictly increasing transformations of $X$ and $Y$ [1, Sec. 2.4].

Let $F_{X}$ and $F_{Y}$ denote the standard Gaussian distribution functions of $X$ and $Y$. Transforming $X$ and $Y$ to $X^{\prime}$ and $Y^{\prime}$ respectively as follow: $\alpha_{X}=X \sigma_{X}+\mu_{X}$ and $\beta_{Y}=Y \sigma_{Y}+\mu_{Y}$ respectively, where $\mu_{X}$ and $\mu_{Y}$ are expected values, $\sigma_{X}$ and $\sigma_{Y}$ are standard derivations of $X^{\prime}$ and $Y^{\prime}$ respectively, and then the standard Gaussian distribution becomes generalized Gaussian distribution. Note that $\alpha_{X}$ and $\beta_{Y}$ are strictly increasing on $\operatorname{Ran} \boldsymbol{X}$ and $\operatorname{Ran} \boldsymbol{Y}$ respectively, and therefore we can determine that the copula defined by two generalized normal distribution is consistent with the copula defined by two standard normal distribution.

\subsection{Bivariate Log-normal Copula}

Log-normal distributions are usually used to model the slow fading channel for communication systems [24]. Let two log-normal pdfs of $x$ and $y$ be $f_{X}(x)=\frac{1}{x \sqrt{2 \pi} \sigma_{X}} \exp \left(-\frac{1}{2}\left(\frac{\log (x)-\mu_{X}}{\sigma_{X}}\right)^{2}\right)$ and $f_{Y}(y)=\frac{1}{y \sqrt{2 \pi} \sigma_{Y}} \exp \left(-\frac{1}{2}\left(\frac{\log (y)-\mu_{Y}}{\sigma_{Y}}\right)^{2}\right)$, respectively. Note that both $\log (x)$ and $\log (y)$ have a normal distribution, where $\mu_{X}$ and $\mu_{Y}$ are the corresponding mathematical expectations and $\sigma_{X}$ and $\sigma_{Y}$ the corresponding standard deviations of the two random variables of $\log (x)$ and $\log (y)$, respectively. Let $F_{X}$ and $F_{Y}$ denote the standard Gaussian distribution functions of $X$ and $Y$, we can respectively transform $X$ to $X^{\prime}$ and $Y$ to $Y^{\prime}$ using $\alpha_{X}=\exp \left(\mu_{X}+X \sigma_{X}\right)$ and $\beta_{Y}=\exp \left(\mu_{Y}+Y \sigma_{Y}\right)$, thus both $X^{\prime}$ and $Y^{\prime}$ satisfy a log-normal distribution. Note that $\alpha_{X}$ and $\beta_{Y}$ are strictly increasing on $\operatorname{RanX}$ and $\operatorname{Ran} \boldsymbol{Y}$, respectively, thus the log-normal copula is consistent with the Gaussian copula. The log-normal copula, with four more parameters $A, B, m, n$ used, was firstly derived in [5], but the author did not point out this equivalence. This does not affect the final definition of log-normal copula function, as we can simply transform $X$ and $Y$ as follows: $\alpha_{X}=\frac{\log (X / A)-m \mu_{X}}{m \sigma_{X}}$ and $\beta_{Y}=\frac{\log (Y / B)-n \mu_{Y}}{n \sigma_{Y}}$. Note that $\alpha_{X}$ and $\beta_{Y}$ are strictly increasing on $\operatorname{Ran} X$ and $\operatorname{Ran} Y$ respectively, and thus the definition of log-normal is invariant.

\subsection{Bivariate Weibull/Rayleigh/Exponential Copula}

The Weibull distribution is usually used for modeling channel fading of communications [22] and wind speed in power systems [26]. Let the two Weibull pdfs be $f_{X}(x)=\frac{\beta_{X}}{\Omega_{X}} x^{\beta_{X}-1} \exp \left(-\frac{x^{\beta_{X}}}{\Omega_{X}}\right)$ and $f_{Y}(y)=\frac{\beta_{Y}}{\Omega_{Y}} y^{\beta_{Y}-1} \exp \left(-\frac{y^{\beta_{Y}}}{\Omega_{Y}}\right)$, respectively, where $\beta_{X} \geq 0$, $\beta_{Y} \geq 0, \Omega_{x}=E\left(x^{\beta_{X}}\right)$ and $\Omega_{Y}=E\left(y^{\beta_{Y}}\right)$, and $E($.$) denotes the operator of mathematical expectation. Then, the associated marginal$ cdfs become $F_{X}(x)=1-\exp \left(-\frac{x^{\beta_{X}}}{\Omega_{X}}\right)$ and $F_{Y}(y)=1-\exp \left(-\frac{y^{\beta_{Y}}}{\Omega_{Y}}\right)$. Thus, the corresponding bivariate Weibull density function is obtained as [22]: 


$$
f_{X Y}(x, y)=\frac{\beta_{X} \beta_{Y} x^{\beta_{X}-1} y^{\beta_{Y}-1}}{\Omega_{X} \Omega_{Y}(1-\rho)} \exp \left[\frac{-1}{(1-\rho)}\left(\frac{x^{\beta_{X}}}{\Omega_{X}}+\frac{y^{\beta_{Y}}}{\Omega_{Y}}\right)\right] I_{0}\left(\frac{2 x^{\frac{\beta_{X}}{2}} y^{\frac{\beta_{Y}}{2}} \sqrt{\rho}}{(1-\rho) \sqrt{\Omega_{X} \Omega_{Y}}}\right)
$$

where $0 \leq \rho<1$ denotes the power correlation coefficient. The modified Bessel functions of the first kind with $n^{\text {th }}$ order are defined as [27, Eq. 9.6.10]:

$$
I_{n}(z)=\left(\frac{z}{2}\right)^{n} \sum_{k=0}^{\infty} \frac{(z / 2)^{2 n}}{k ! \Gamma(n+k+1)}
$$

where $\Gamma($.$) is the Gamma function [27, eq. 6.1.1 and 6.1.6]. Note that the inverse marginal cdfs are obtained as$ $x=F_{X}^{-1}(u)=\left[-\Omega_{X} \ln (1-u)\right]^{\frac{1}{\beta_{X}}}$ and $y=F_{Y}^{-1}(v)=\left[-\Omega_{Y} \ln (1-v)\right]^{\frac{1}{\beta_{Y}}}$.

As a result, the Weibull copula can be rewritten as:

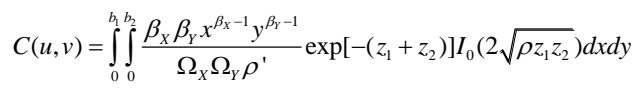

where we have $b_{1}=\sqrt{-\Omega_{X} \ln (1-u)}$ and $b_{2}=\sqrt{-\Omega_{Y} \ln (1-v)} ; z_{1}=\left(\rho^{\prime} \Omega_{X}\right)^{-1} x^{\beta_{X}} \in\left[0, w_{1}\right]$ where $w_{1}=-\log (1-u) / \rho^{\prime}$, and $z_{2}=\left(\rho^{\prime} \Omega_{Y}\right)^{-1} y^{\beta_{Y}} \in\left[0, w_{2}\right]$ where $w_{2}=-\log (1-v) / \rho^{\prime}$ and $\rho^{\prime}=1-\rho$.

Since the associated Jacobean determinant is determined as $\left|\begin{array}{ll}\partial x / \partial z_{1} & \partial x / \partial z_{2} \\ \partial y / \partial z_{1} & \partial y / \partial z_{2}\end{array}\right|=\frac{(1-\rho)^{2} \Omega_{X} \Omega_{Y}}{\beta_{X} \beta_{Y} x^{\beta_{X}-1} y^{\beta_{Y}-1}}$. Consequently, (9) can be re-written as:

$$
C(u, v)=\rho^{\prime} \int_{0}^{a_{1} a_{2}} \exp \left(-z_{1}-z_{2}\right) I_{0}\left(2 \sqrt{\rho z_{1} z_{2}}\right) d z_{1} z_{2}
$$

where we have $a_{1}=-\ln (1-u) / \rho^{\prime}$ and $a_{2}=-\ln (1-v) / \rho^{\prime}$. Consequently, we can find that $\rho$ is the only parameter for Weibull copula.

Next, we will discuss the case of exponential copula. Consider as an example, a single-server queuing system, as a common assumption, the inter-arrival time and the service time satisfy an exponential distribution. Downton's bivariate exponential distribution is a suitable choice for the queuing system with infinitely servers [2, Sec. 5.14].

Let two marginal exponential probability density functions be $f_{X}(x)=\lambda \exp (-\lambda x)$ and $f_{Y}(y)=\mu \exp (-\mu y)$, where $x \geq 0, y \geq 0$, $\lambda \geq 0$ and $\mu \geq 0$. The expectations of $x$ and $y$ can be decided as $1 / \lambda$ and $1 / \mu$, respectively. Thus, their two marginal cdfs can be determined as $F_{X}(x)=1-\exp (-\lambda x)$ and $F_{Y}(y)=1-\exp (-\mu y)$, respectively. Finally, the associated Downton's bivariate exponential distribution has a pdf as [2, Eq. 5.5.7] 


$$
f_{X Y}(x, y)=\frac{\lambda \mu}{1-\rho} \exp \left(-\frac{\lambda x}{1-\rho}-\frac{\mu y}{1-\rho}\right) I_{0}\left(\frac{2}{1-\rho} \sqrt{\rho \lambda \mu x y}\right)
$$

where $\rho$ is the correlation coefficient of $x$ and $y$ satisfying $0 \leq \rho<1$, and $I_{0}($.$) is the modified Bessel function of the first kind with$ $0^{\text {th }}$ order. Clearly, $x$ and $y$ are independent only if $\rho=0$.

From Eq. (10), we have found that $\rho$ is the only parameter for Weibull copula. This implies that we can define the Weibull copula by two Weibull distributions with arbitrary parameters $\beta_{X}$ and $\beta_{Y}$ respectively. As a matter of fact, the Weibull distribution becomes exponential distribution when $\beta_{X}=1$ and $\beta_{Y}=1$, while this will not affect the final definition of Weibull copula. Therefore, we can determine that the exponential copula equals to the Weibull copula.

Next, we will discuss the Rayleigh copula function. The Rayleigh distribution is frequently used in communication domain to model the received signal amplitudes in urban and suburban areas [23]. Let two marginal Rayleigh density functions be $f_{X}(x)=\frac{2 x}{\Omega_{X}} \exp \left(-\frac{x^{2}}{\Omega_{X}}\right)$ and $f_{Y}(y)=\frac{2 y}{\Omega_{Y}} \exp \left(-\frac{y^{2}}{\Omega_{Y}}\right)$, where $x \geq 0, y \geq 0$. The associated two marginal cdfs are $F_{X}(x)=1-\exp \left(-\frac{x^{2}}{\Omega_{X}}\right)$ and $F_{Y}(y)=1-\exp \left(-\frac{y^{2}}{\Omega_{Y}}\right)$, where $\Omega_{x}=E\left(x^{2}\right)$ and $\Omega_{Y}=E\left(y^{2}\right)$, and $E($.$) denotes the operator of mathematical expectation. The$ corresponding bivariate Rayleigh density function can be derived as [25, Eq. 6.2]:

$$
f_{X Y}(x, y)=\frac{4 x y}{\Omega_{X} \Omega_{Y} \rho^{\prime}} \exp \left[\frac{-1}{\rho^{\prime}}\left(\frac{x^{2}}{\Omega_{X}}+\frac{y^{2}}{\Omega_{Y}}\right)\right] I_{0}\left(\frac{2 x y}{\rho^{\prime}} \sqrt{\frac{\rho}{\Omega_{X} \Omega_{Y}}}\right)
$$

where $0 \leq \rho<1$ is the power correlation coefficient of $x$ and $y$.

We have proven that $\rho$ is the only parameter for Weibull copula in Eq. (10), and therefore we can define the Weibull copula by two Weibull distribution with arbitrary parameters $\beta_{X}$ and $\beta_{Y}$ respectively. We can simply let $\beta_{X}=2$ and $\beta_{Y}=2$, then the Weibull distribution becomes Rayleigh distribution, and this will not change the final definition of Weibull copula. Consequently, we can determine that the Rayleigh copula equals to the Weibull copula as well.

To compute the bivariate Weibull/exponential/Rayleigh copula, the following formula can be used to reduce the double integral to a single integral [28]:

$$
(1-\rho) \int_{0}^{x} \int_{0}^{y} e^{-(s+t)} I_{0}(2 \sqrt{\rho s t}) d s d t=\left(1-e^{\rho y-y}\right)+e^{\rho y-y} K(\rho y, x)-e^{\rho x-x} K(y, \rho x)
$$

where $\rho \geq 0$ and $K(x, y)=e^{-y} \int_{0}^{x} e^{-s} I_{0}(2 \sqrt{s y}) d s$.

After algebraic manipulation, (10) can be replaced by the following formula that only includes a single integral for simplicity: 


$$
C(u, v)=1+e^{\rho a_{2}-a_{2}}\left[\int_{0}^{\rho a_{2}} e^{-s} I_{0}\left(2 \sqrt{a_{1} s}\right) d s-1\right]-e^{-a_{1}} \int_{0}^{a_{2}} e^{-s} I_{0}\left(2 \sqrt{\rho a_{1} s}\right) d s
$$

As $K(x, y)=e^{-y} \int_{0}^{x} e^{-s} I_{0}(2 \sqrt{s y}) d s . K(x, y)$ can also be expressed by Marcum's $Q$ function as follows [29]:

$$
Q_{M}(a, b)=\int_{b}^{\infty} x\left(\frac{x}{a}\right)^{M-1} e^{-\frac{x^{2}+a^{2}}{2}} I_{M-1}(a x) d x
$$

Let $Q_{I}(a, b)$ denote the special case of Marcum's $Q$ function with $M=1$ and $Q_{1}(a, b)=\int_{b}^{\infty} x e^{-\frac{x^{2}+a^{2}}{2}} I_{0}(a x) d x$, we have

$$
\int_{0}^{b} x e^{-\left(x^{2}+p^{2}\right) / 2} I_{0}(a x) d x=\left[1-Q_{1}(a / p, b p)\right] e^{-\frac{a^{2}}{2 p^{2}}} / p^{2}
$$

After algebraic manipulation, we can derive the relationship between $K(x, y)$ and $Q_{1}(x, y)$ as $K(x, y)=1-Q_{1}(\sqrt{2 y}, \sqrt{2 x})$. As a result, Eq. (10) and (14) can be rewritten as [5, Eq. (4)]:

$$
\begin{aligned}
& C(u, v)=1-(1-v) a_{3}-(1-u)\left(1-a_{3}\right) \\
& a_{3}=Q_{1}\left(\sqrt{2 a_{1}}, \sqrt{2 a_{2}}\right)
\end{aligned}
$$

Apparently, this has validated that Eq. (14) is a new representation of bivariate Rayleigh copula that only requires a single integral.

The computation results of bivariate Weibull/Rayleigh/Exponential copula with the parameter $\rho=0.3$ and $\rho=0.8$ are shown in Fig. 1(a) and Fig. 1(c), respectively, and their contour plots are shown in Fig. 1(b) and Fig. 1(d) respectively, which have validated the properties of the copula as defined in Eq. (3) and Eq. (4). In the plots of generated contours, we can clearly find that the isolines have the following characteristics. Firstly, on each separate isoline, its curvature increases when the difference between u and v decreases, which reaches the maximum when $u=v$. Secondly, the isolines have lower curvaturess when $(u+v)$ is large but higher curvaturess when $(\mathrm{u}+\mathrm{v})$ is small. Thirdly, when $\rho$ increases from 0 to 1 , the maximum curvature achieved in each isoline increases, though the remaining part of isolines becomes flatter than those of a smaller $\rho$. In an extreme case when $\rho=0$, perfectly parallel straight lines with the slope of -45 degrees will be produced in the contour plot. Moreover, the perpendicular lines with corners lying on the diagonal will appear when $\rho=1$.

\subsection{Bivariate Nakagami-m Copula}

Nakagami-m distribution is frequently used to model fading in wireless environment [23]. Considering two marginal Nakagami-m pdfs of random variables $x$ and $y$ as $f_{X}(x)=\frac{2 m^{m} x^{2 m-1}}{\Gamma(m) \Omega_{X}^{m}} \exp \left(-\frac{m x^{2}}{\Omega_{X}}\right)$ and $f_{Y}(y)=\frac{2 m^{m} y^{2 m-1}}{\Gamma(m) \Omega_{Y}^{m}} \exp \left(-\frac{m y^{2}}{\Omega_{Y}}\right)$, respectively, 
where $x>0$ and $y>0$. The marginal cdfs can be determined as $F_{X}(x)=P\left(m, \frac{m x^{2}}{\Omega_{X}}\right)$ and $F_{Y}(y)=P\left(m, \frac{m y^{2}}{\Omega_{Y}}\right)$, where $P($.$) is the regulated$ lower incomplete Gamma function being defined as $P(a, z)=\frac{\gamma(a, z)}{\Gamma(a)}=\frac{1}{\Gamma(a)} \int_{0}^{z} t^{a-1} \exp (-t) d t[27$, eq. (6.5.1)], and $\gamma($.$) is the lower$ incomplete Gamma function defined as $\gamma(a, z)=\int_{0}^{z} t^{a-1} \exp (-t) d t$ [27, eq. (6.5.2)]. The associated joint pdf can then be derived as [6]:

$$
f_{X Y}(x, y)=\frac{4 \rho m(1-\rho)^{-1}(m x y)^{m}}{\Gamma(m)\left(\sqrt{\rho \Omega_{X} \Omega_{Y}}\right)^{m+1}} \exp \left(-z_{1}-z_{2}\right) I_{m-1}\left(2 \sqrt{\rho z_{1} z_{2}}\right)
$$

where $0 \leq \rho<1$ is the power correlation coefficient of $x$ and $y, \rho^{\prime}=1-\rho, z_{1}=\frac{m x^{2}}{\rho^{\prime} \Omega_{X}}$ and $z_{2}=\frac{m y^{2}}{\rho^{\prime} \Omega_{Y}}$. The copula function of the bivariate Nakagami-m distribution has been defined with two parameters $\rho$ and $m$ as [6]:

$$
C(u, v)=\frac{(1-\rho)^{m}}{\Gamma(m)} \sum_{k=0}^{\infty}\left[\frac{\rho^{k} \Gamma(m+k)}{2 k !}\right] P\left[m+k, \frac{P^{-1}(m, u)}{1-\rho}\right] P\left[m+k, \frac{P^{-1}(m, v)}{1-\rho}\right]
$$

The computation results of bivariate Nakagami-m copula with the parameter $\rho=0.3 ; m=2, \rho=0.8 ; m=2$ and $\rho=0.8 ; m=5$ are shown in Fig. 2 (a), Fig. 2 (c) and Fig. 2(e) respectively, and their contour plots are shown in Fig. 2. (b), Fig. 2(d) and Fig. 2(f) respectively, which have validated the properties of the copula defined in Eq. (3) and Eq. (4). Overall, the generated isolines have similar characteristics as those in the results of bivariate Weibull/Rayleigh/Exponential copula functions: the curvature decrease when $\mathrm{u}+\mathrm{v}$ increases and reaches the extreme when $\mathrm{u}=\mathrm{v}$; When $\rho$ increases, the maximum curvature achieved also increases. Again if the parameter $\rho$ is set to 0 , perfectly parallel straight lines with the slope of -45 degrees will be shown in the contour plot. If the parameter $\rho$ is set to 1 , the perpendicular lines with corners lying on the diagonal will be generated. In addition, the contours seem insensitive to the parameter $m$ when its value is changed from 2 to 5 .

\subsection{Bivariate Rician Copula}

Rician distribution is widely used to model the amplitude fluctuations of received signals from different multipath fading [23].

Considering two marginal Rician pdfs of random variables $x$ and $y$ as $f_{X}(x)=\frac{x}{\sigma^{2}} \exp \left(-\frac{x^{2}+a^{2}}{2 \sigma^{2}}\right) I_{0}\left(\frac{a x}{\sigma^{2}}\right)$ and $f_{Y}(y)=\frac{y}{\sigma^{2}} \exp \left(-\frac{y^{2}+a^{2}}{2 \sigma^{2}}\right) I_{0}\left(\frac{a y}{\sigma^{2}}\right)$, respectively, where $x \geq 0$ and $y \geq 0$, and $a$ is the non-centrality parameter. When $a=0$, the Rician distribution degrades to the Rayleigh distribution. The marginal cdfs of $x$ and $y$ are $F_{X}(x)=1-Q_{1}\left(\frac{a}{\sigma}, \frac{x}{\sigma}\right)$ and $F_{Y}(y)=1-Q_{1}\left(\frac{a}{\sigma}, \frac{y}{\sigma}\right)$, respectively [30, Eq. (2.18)], and the associated bivariate Rician pdf is given in [30], [31] as 


$$
f_{X Y}(x, y)=\frac{x y}{\left(1-\rho^{2}\right) \sigma^{4}} \exp \left(-\frac{x^{2}+y^{2}+2(1-\rho) a^{2}}{2\left(1-\rho^{2}\right) \sigma^{2}}\right) \sum_{k=0}^{+\infty} \varepsilon_{k} I_{k}\left(\frac{x y \rho}{\left(1-\rho^{2}\right) \sigma^{2}}\right) I_{k}\left(\frac{a x}{(1+\rho) \sigma^{2}}\right) I_{k}\left(\frac{a y}{(1+\rho) \sigma^{2}}\right)
$$

where $0 \leq \rho<1$ is the correlation coefficient of $x$ and $y$ and $\varepsilon$ is the Neumann factor defined as $\varepsilon_{m}=\left\{\begin{array}{ll}1 & m=0 \\ 2 & m>0\end{array}[\right.$ [30]. The associated bivariate Rican copula can be written as:

$$
C(u, v)=\int_{0}^{x_{0} y_{0}} \frac{x y}{0} \frac{x y}{\left(1-\rho^{2}\right) \sigma^{4}} \exp \left(-\frac{x^{2}+y^{2}+2(1-\rho) a^{2}}{2\left(1-\rho^{2}\right) \sigma^{2}}\right) \sum_{k=0}^{+\infty} \varepsilon_{k} I_{k}\left(\frac{x y \rho}{\left(1-\rho^{2}\right) \sigma^{2}}\right) I_{k}\left(\frac{a x}{(1+\rho) \sigma^{2}}\right) I_{k}\left(\frac{a y}{(1+\rho) \sigma^{2}}\right) d x d y
$$

where $x_{0}=\sigma Q_{1}^{-1}(a / \sigma, 1-u)$ and $y_{0}=\sigma Q_{1}^{-1}(a / \sigma, 1-v)$.

Let $z_{1}=x \sigma^{-1}, z_{2}=y \sigma^{-1}$ and $z=a \sigma^{-1}$, thus we have $0 \leq z_{1} \leq Q_{1}^{-1}(z, 1-u)$ and $0 \leq z_{2} \leq Q_{1}^{-1}(z, 1-v)$. The associated Jacobean determinant becomes $\left|\begin{array}{ll}\partial x / \partial z_{1} & \partial x / \partial z_{2} \\ \partial y / \partial z_{1} & \partial y / \partial z_{2}\end{array}\right|=\sigma^{2}$. As a result, the bivariate Rician copula function in Eq. (32) can be derived as:

$$
C(u, v)=\int_{0}^{b_{1} b_{2}} \frac{z_{1} z_{2}}{1-\rho^{2}} \exp \left(-\frac{z_{1}^{2}+z_{2}^{2}+2(1-\rho) z^{2}}{2\left(1-\rho^{2}\right)}\right) \sum_{k=0}^{+\infty} \varepsilon_{k} I_{k}\left(\frac{\rho z_{1} z_{2}}{1-\rho^{2}}\right) I_{k}\left(\frac{z_{1} z}{1+\rho}\right) I_{k}\left(\frac{z_{2} z}{1+\rho}\right) d z_{1} d z_{2}
$$

where $b_{1}=Q_{1}^{-1}(z, 1-u)$ and $b_{2}=Q_{1}^{-1}(z, 1-v)$.

Note that the Rician factor $k$ can be defined as $K=\frac{a^{2}}{2 \sigma^{2}}$, and thus $z=\sqrt{2 K}$. Therefore, the bivariate Rician copula function can also be written as:

$$
C(u, v)=\int_{0}^{b_{1} b_{2}} \int_{0}^{\frac{z_{1}}{1-\rho_{2}}} \exp \left(-\frac{z_{1}^{2}+z_{2}^{2}+4(1-\rho) K}{2\left(1-\rho^{2}\right)}\right) \sum_{k=0}^{+\infty} \varepsilon_{k} I_{k}\left(\frac{\rho z_{1} z_{2}}{1-\rho^{2}}\right) I_{k}\left(\frac{z_{1} \sqrt{2 K}}{1+\rho}\right) I_{k}\left(\frac{z_{2} \sqrt{2 K}}{1+\rho}\right) d z_{1} d z_{2}
$$

where $b_{1}=Q_{1}^{-1}(\sqrt{2 K}, 1-u)$ and $b_{2}=Q_{1}^{-1}(\sqrt{2 K}, 1-v)$.

Also note that the bivariate Rician copula relies only on the parameters $z$ (or $K$ ) and $\rho$. Also note that Marcum's $Q$ function $Q_{M}(a, b)$ in Eq. (15) monotonically decreases from 1 to 0 as $b$ varies from 0 to $\infty$, hence the bisection method [32] has been proposed for evaluating the inverse Marcum's function in [33]. The form of the bivariate Rician copula is complicated, but it can be readily computed by the numerical integral method [32]. Note that the bivariate Rician distribution function can be represented by the infinite series representation by expanding the modified first kind of Bessel function as follows [31], [34]:

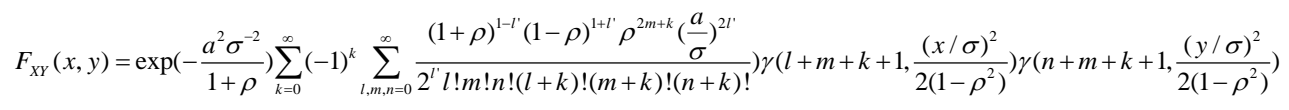


where $l^{\prime}=l+n+k$, and $\gamma($.$) is the lower incomplete Gamma function defined as [27, Eq. (6.5.2)] as \gamma(a, z)=\int_{0}^{z} t^{a-1} \exp (-t) d t$.

Let $u=F_{X}(x)$, and $v=F_{Y}(y)$, then $\frac{x}{\sigma}=Q_{1}^{-1}(z, 1-u)$ and $\frac{y}{\sigma}=Q_{1}^{-1}(z, 1-v)$ where $z=\frac{a}{\sigma}$, then we can derive the bivariate Rician copula function $\mathrm{C}(u, v)$ expressed by infinite series from $F_{X Y}(x, y)$ as:

$$
C(u, v)=\exp \left(\frac{-z^{2}}{1+\rho}\right) \sum_{k=0}^{\infty} \varepsilon_{k}(-1)^{k} \sum_{l, m, n=0}^{\infty} \frac{(1+\rho)^{1-l^{\prime}}(1-\rho)^{1+l^{\prime}} \rho^{2 m+k} z^{2 l^{\prime}}}{2^{l} l ! m ! n !(l+k) !(m+k) !(n+k) !} \gamma\left(l+m+k+1, \frac{\left[Q_{1}^{-1}(z, 1-u)\right]^{2}}{2\left(1-\rho^{2}\right)}\right) \gamma\left(n+m+k+1, \frac{\left[Q_{1}^{-1}(z, 1-v)\right]^{2}}{2\left(1-\rho^{2}\right)}\right)
$$

Although Eq. (25) can be directly used to compute bivariate Rician copula, it suffers from the vast amount of computation needed in resolving the inverse Marcum $Q$ function. Assume we need to determine the result of bivariate Rician copula $C\left(u_{1}, v_{l}\right)$ with parameters $\rho_{1}$ and $z_{1}$, we need firstly calculate $Q_{1}^{-1}\left(z_{1}, 1-u_{1}\right)$ and $Q_{1}^{-1}\left(z_{1}, 1-v_{1}\right)$ for avoiding resolving the inverse Marcum $\mathrm{Q}$ function in the incomplete Gamma function of the infinite summation as they are irrelevant to $k, l, m$ and $n$. Alternatively, Eq. (24) can be applied to compute bivariate Rician copula as follows. Assume we need to calculate the result of bivariate Rician copula $C\left(u_{1}, v_{l}\right)$ with parameters $\rho_{l}$ and $z_{1}$, we can simply get $x_{l}$ and $y_{l}$ from $u_{1}=1-Q_{1}\left(z_{1}, x_{1}\right)$ and $v_{1}=1-Q_{1}\left(z_{1}, y_{1}\right)$. Next, we can substitute $x_{l}$ and $y_{1}$ to Eq. (24) and also let $a=z_{1}$ to compute the value of $C\left(u_{l}, v_{l}\right)$ with parameters $\rho_{l}$ and $z_{l}$. In this way the difficulty in computing the inverse Marcum Q function in the infinite summation in Eq. (25) is also avoided though the whole process is still quite time consuming. The truncation error upper bound for the infinite series in (24) is given in [34], and the truncation is relevant to $u, v, \rho$ and $z$. It also has been pointed out that computing the nested infinite summations is usually tedious even if they are truncated, since the calculation time and accuracy solely depend on the available computing power [34]. In addition, Eq. (22) and (23) can also be used to determine the bivariate Rician copula using numerical integration [32]. This method is adopted in this paper,

since the former two methods using either Eq. (25) or Eq. (24) are too time consuming to compute the bivariate Rician distribution with 30 different values of $u$ and $v$.

The computation results of bivariate Rician copula with the parameter $\rho=0.3 ; z=0.5, \rho=0.3 ; z=2$ and $=0.8 ; z=2$ are shown in Fig. 3(a), Fig. 3(c) and Fig. 3(e) respectively, and their contour plots are shown in Fig. 3(b), Fig. 3(d) and Fig. 3(f) respectively, which have validated the properties of the copula defined in Eq. (3) and Eq. (4). In this group of plots, we can find that the generated contours are insensitive to the parameter $z$. On the other hand, the isolines feature the same characteristics as the previous two cases when $u, v$ and $\rho$ change.

\section{COPULA DENSITY FUnCTIONS}

In practical applications, copula density functions are often more frequently used than the copula function itself, e.g., copula 
density has been applied to estimate mutual information [7]. From the copula function, its density function can be derived by definition in Eq. (26) below, where specific density functions for various copulas are discussed in detail in this section.

$$
c(u, v)=\frac{\partial^{2} C(u, v)}{\partial u \partial v}=\frac{\partial^{2} C\left[F_{X}(x), F_{Y}(y)\right]}{\partial F_{X}(x) \partial F_{Y}(y)}
$$

\subsection{Bivariate Log-normal Copula}

We have proved in Section (2.2) that the bivariate log-normal copula and bivariate Gaussian copula are equivalent. Therefore, the density functions of bivariate log-normal copula and bivariate normal copula are also equivalent and can be written as [7]:

$$
c(u, v)=\frac{1}{\sqrt{1-\rho^{2}}} \exp \left(\frac{2 \rho \Phi^{-1}(u) \Phi^{-1}(v)-\rho^{2}\left\{\left[\Phi^{-1}(u)\right]^{2}+\left[\Phi^{-1}(v)\right]^{2}\right\}}{2\left(1-\rho^{2}\right)}\right)
$$

where $\Phi($.$) represents the cdf of standard Gaussian distribution.$

\subsection{Bivariate exponential, Rayleigh and Weibull copulas}

Since bivariate exponential, Rayleigh and Weibull copulas have been proved to be equivalent, here only bivariate exponential copula is considered in deriving the density function. According to the Eq. (11), bivariate exponential copula has the form as

$$
C(u, v)=\int_{0}^{\frac{\log (1-u)}{-\lambda}} \int_{0}^{\frac{\log (1-v)}{-\mu}} \frac{\lambda \mu}{1-\rho} \exp \left(-\frac{\lambda x+\mu y}{1-\rho}\right) I_{0}\left(\frac{2 \sqrt{\rho \lambda \mu x y}}{1-\rho}\right) d x d y
$$

where $x=-\ln (1-u) / \lambda$ and $y=-\ln (1-v) / \mu$. Note that $\frac{1}{F_{X}{ }^{\prime}(x)}=\frac{1}{\lambda \exp (-\lambda x)}$ and $\frac{1}{F_{Y}{ }^{\prime}(y)}=\frac{1}{\mu \exp (-\mu y)}$. After algebraic manipulation, we can derive the copula density function as

$$
c(u, v)=\frac{1}{1-\rho} \exp \left(\frac{\rho\left[\ln \left(u^{\prime}\right)+\ln \left(v^{\prime}\right)\right]}{1-\rho}\right) I_{0}\left(\frac{2 \sqrt{\rho \ln \left(u^{\prime}\right) \ln \left(v^{\prime}\right)}}{1-\rho}\right)
$$

where $u^{\prime}=1-u$ and $v^{\prime}=1-v$.

From (28), it also can be found again that the bivariate exponential (and also Rayleigh and Weibull) copula rely only on the parameter $\rho$. Plots of the bivariate exponential (also Rayleigh and Weibull) copula density functions with $\rho=0.3$ and $\rho=0.8$ are given in Fig. 4(a) and Fig. 4(b) respectively. If the parameter $\rho$ is set to 0 , we have $c(u, v)=1$ for arbitrary values of $u$ and $v$. When the parameter $\rho$ increases from 0 to 1 , higher values of $c(u, v)$ occur when $u$ and $v$ are close. On the other hand, $c(u, v)$ decreases and approaches to 0 when the values of $\mathrm{u}$ and $\mathrm{v}$ are dissimilar.

\subsection{Bivariate Nakagami-m copula}

The copula function of bivariate Nakagami-m distribution has been defined in [5] as 


$$
C(u, v)=\frac{(1-\rho)^{m}}{\Gamma(m)} \sum_{k=0}^{\infty}\left[\frac{\rho^{k} \Gamma(m+k)}{2 k !}\right] P\left[m+k, \frac{P^{-1}(m, u)}{1-\rho}\right] P\left[m+k, \frac{P^{-1}(m, v)}{1-\rho}\right]
$$

where $\frac{m x^{2}}{\Omega_{X}}=P^{-1}(m, u)$ and $\frac{m y^{2}}{\Omega_{Y}}=P^{-1}(m, v)$. Note that the partial derivatives of the regulated lower incomplete Gamma function have been evaluated in [27, eq. (6.5.25)] as

$$
\frac{\partial P(a, z)}{\partial z}=\frac{e^{-z} z^{a-1}}{\Gamma(a)}
$$

Thus we have $\frac{1}{F_{X}{ }^{\prime}(x)}=\frac{\Gamma(m) \Omega_{X}}{2 m x\left[P^{-1}(m, u)\right]^{m-1} \exp \left[P^{-1}(m, u)\right]}$ and $\frac{1}{F_{Y}{ }^{\prime}(y)}=\frac{\Gamma(m) \Omega_{X}}{2 m y\left[P^{-1}(m, v)\right]^{m-1} \exp \left[P^{-1}(m, v)\right]}$. After algebraic manipulation, we can derive the copula density of Nakagami-m distribution as

$$
c(u, v)=\frac{\Gamma(m)(\widehat{u} \widehat{v})^{-(m-1) / 2}}{(1-\rho) \rho^{(m-1) / 2}} \exp \left(\frac{-\rho(\widehat{u}+\widehat{v})}{1-\rho}\right) I_{m-1}\left(\frac{2 \sqrt{\rho \widehat{u} \widehat{v}}}{1-\rho}\right)
$$

where $\widehat{u}=P^{-1}(m, u), \widehat{v}=P^{-1}(m, v), \Gamma($.$) is the Gamma function [27, Eq. 6.1.1 and 6.1.6] and P($.$) is regulated lower incomplete$ Gamma function. From (40), again it validates that the bivariate Nakagami-m copula rely only on the parameters $\rho$ and $m$.

Plots of the bivariate Nakagami-m copula density with parameter $\rho=0.3 ; m=2, \rho=0.8 ; m=2$ and $=0.8 ; m=5$ are shown in Fig. 5 (a), Fig 5(b) and Fig. 5(c) respectively. If the parameter $\rho$ is close to 0 , the value of $c(u, v)$ approaches 1 for arbitrary values of $u$ and $v$. If the parameter $\rho$ is close to 1 , higher values of $c(u, v)$ occur when the values of $u$ and $v$ are close to each other; $c(u, v)$ decreases and approaches to 0 when the difference of $u$ and $v$ increases. If the parameter is fixed, for small values of $u$ and $v, c(u, v)$ increases when $m$ is increasing; whilst for high values of $u$ and $v, \mathrm{c}(\mathrm{u}, \mathrm{v})$ decreases when the parameter $m$ increases.

\subsection{Bivariate Rician copula}

The copula function of bivariate Rician distribution has been defined in (32) as:

$$
C(u, v)=\int_{0}^{x_{0} y_{0}} \frac{x y}{\left(1-\rho^{2}\right) \sigma^{4}} \exp \left(-\frac{x^{2}+y^{2}+2(1-\rho) a^{2}}{2\left(1-\rho^{2}\right) \sigma^{2}}\right) \sum_{k=0}^{+\infty} \varepsilon_{k} I_{k}\left(\frac{x y \rho}{\left(1-\rho^{2}\right) \sigma^{2}}\right) I_{k}\left(\frac{a x}{(1+\rho) \sigma^{2}}\right) I_{k}\left(\frac{a y}{(1+\rho) \sigma^{2}}\right) d x d y
$$

where $x_{0}=\sigma Q_{1}^{-1}(a / \sigma, 1-u)$ and $y_{0}=\sigma Q_{1}^{-1}(a / \sigma, 1-v)$.

Let $z_{1}=\frac{x}{\sigma} \in\left[0, a_{1}\right], z_{2}=\frac{y}{\sigma} \in\left[0, a_{2}\right]$ and $z=\frac{a}{\sigma}$, where $a_{1}=Q_{1}^{-1}(z, 1-u)$ and $a_{2}=Q_{1}^{-1}(z, 1-v)$. Note that the partial derivative of Marcum's $Q$ function can be represented as [35]

$$
\frac{\partial Q_{1}(a, b)}{\partial b}=-a I_{0}(a b) \exp \left(-\frac{a^{2}+b^{2}}{2}\right)
$$


Therefore, we have $\frac{1}{F_{X}{ }^{\prime}(x)}=\frac{\sigma \exp \left\{-\frac{1}{2}\left[Q_{1}^{-1}(z, 1-u)\right]^{2}+(z)^{2}\right\}}{Q_{1}^{-1}(z, 1-u) I_{0}\left(z, \frac{x}{\sigma}\right)}$ and $\frac{1}{F_{Y}{ }^{\prime}(y)}=\frac{\sigma \exp \left\{-\frac{1}{2}\left[Q_{1}^{-1}(z, 1-v)\right]^{2}+z^{2}\right\}}{Q_{1}^{-1}(z, 1-v) I_{0}\left(z, \frac{y}{\sigma}\right)}$. Finally, we can derive the density function of bivariate Rician copula density function as:

$$
c(u, v)=\frac{\exp \left(-\frac{\rho^{2}\left(a_{1}^{2}+a_{2}^{2}\right)+2 \rho(\rho-1) z^{2}}{2\left(1-\rho^{2}\right)}\right)}{\left(1-\rho^{2}\right) I_{0}\left(z, a_{1}\right) I_{0}\left(z, a_{2}\right)} \sum_{k=0}^{\infty} \varepsilon_{k} I_{k}\left(\frac{\rho a_{1} a_{2}}{1-\rho^{2}}\right) I_{k}\left(\frac{a_{1} z}{1+\rho}\right) I_{k}\left(\frac{a_{2} z}{1+\rho}\right)
$$

From (42), it can be found again that the bivariate Rician copula rely only on the parameters $\rho$ and $z$. Plots of the bivariate of Rician copula density with parameter $\rho=0.3 ; z=0.5, \rho=0.8 ; z=0.5$ and $=0.8 ; z=2$ are shown in Fig. 6 (a), Fig 6(b) and Fig. 6(c) respectively. If the parameter $\rho$ is close to $0, c(u, v)$ is close to 1 for arbitrary values of $u$ and $v$. If the parameter $\rho$ is close to 1 , higher values of $c(u, v)$ occur when $u$ and $v$ are close to each other; $c(u, v)$ decreases and approaches to 0 when the difference between $u$ and $v$ increases. If the parameter $\rho$ is fixed, for small values of $u$ and $v$, the values of $c(u, v)$ increases when $m$ increases; whilst for high values of $u$ and $v, c(u, v)$ will decrease even $m$ is increasing.

\section{CONCLUSIONS}

Although copulas have been successfully applied for many signal/image processing and communication problems, their applications are constrained due to the lack of suitable copulas and in-depth analysis of these functions. As a result, we focus on extension and generalization of copulas. Firstly, copula functions associated with the bivariate exponential, Weibull and Rician distributions are derived. Secondly, a new representation of bivariate Rayleigh copula with a single parameter, using single integral, is also derived. Thirdly, we have proved that log-normal copula is equivalent to Gaussian copula that has only one parameter, and Rayleigh copula is equivalent to exponential and Weibull copula that also has only one parameter. The Rician copula is derived to have two parameters. Fourthly, all the modified Bessel function or incomplete Gamma function involved double integrals in the copula functions are represented by single integral or infinite series. Finally, the density functions of log-normal, exponential, Rayleigh, Weibull, Nakagami-m and Rician copulas are also derived. Since only bivariate copulas are focused in this paper, multivariate cases will be studied in the future for further improved applicability. Relevant details on random variables generation of these new copulas and criteria for optimal copula selection as well as simulation and case studies will be discussed in the Part II of the paper.

\section{REFERENCES}

[1] R. B. Nelsen, An Introduction to Copulas, 2nd ed., New York, Springer Verlag, 2006. 
[2] N. Balakrishnan and C.D. Lai, Continuous bivariate distribution, 2nd ed., New York: Springer-Verlag, 2009.

[3] U. Cherubini, E. Luciano and W. Vecchiato, Copula Methods in Finance, New York : John Wiley \& Sons, 2004.

[4] T.S. Durrani and X. Zeng, "Copulas for bivariate probability distributions”, Electronics Letters, Vol. 43, No. 4, pp. 248 - $249,2007$.

[5] X. Liu "Copulas of bivariate Rayleigh and log-normal distributions", Electronics Letters, Vol. 46, No. 25, pp. 1669 - 1671, 2010.

[6] X. Liu “Copula of bivariate Nakagami-m distribution”, Electronics Letters, Vol.47, No.5, pp.343 - 345, 2011.

[7] X. Zeng and T.S Durrani, "Estimation of mutual information using copula density function”, Electronics Letters, Vol. 47, No. 8, pp. 493 - 494, 2011.

[8] B. Storvik, G., Storvik and R. Fjortoft, “On the Combination of Multisensor Data Using Meta-Gaussian Distributions”, IEEE Transactions on Geoscience and Remote Sensing, Vol. 47, No.7, Part: 2, pp. 2372 - 2379, 2009.

[9] S. C. Dass, Y. Zhu and A. K. Jain, "Validating a Biometric Authentication System: Sample Size Requirements", IEEE Transactions on Pattern Analysis and Machine Intelligence, Vol. 28, No. 12, pp. 1902 - 1319, 2006.

[10] G., Papaefthymiou and D. Kurowicka, "Using Copulas for Modeling Stochastic Dependence in Power System Uncertainty Analysis", IEEE Transactions on Power Systems, Vol. 24, No. 1, pp. 40 - 49, 2009.

[11] N.J.B, Brunel, J. Lapuyade-Lahorgue and W. Pieczynski, “Modeling and Unsupervised Classification of Multivariate Hidden Markov Chains with Copulas”, IEEE Transactions on Automatic Control, Vol. 55, No. 2, pp. 338 - 349, 2010.

[12] A., Sundaresan, P.K. Varshney and N.S.V. Rao, "Copula-Based Fusion of Correlated Decisions", IEEE Transactions on Aerospace and Electronic Systems, Vol. 47 , No. 1, pp. $454-471,2011$.

[13] G., Mercier, G., Moser and S.B. Serpico, "Conditional Copulas for Change Detection in Heterogeneous Remote Sensing Images”, IEEE Transactions on Geoscience and Remote Sensing, Vol. 46, No. 5, pp. 1428 - 1441, 2008.

[14] A. Sundaresan and P.K. Varshney, "Estimation of a Random Signal Source Based on Correlated Sensor Observations", IEEE Transactions on Signal Processing, pp. $787-799,2011$.

[15] S.G, Iyengar, P.K Varshney and T. Damarla, “A Parametric Copula-Based Framework for Hypothesis Testing Using Heterogeneous Data”, IEEE Transactions on Signal Processing, Vol. 59, No. 5, pp. 2308 - 2319, 2011.

[16] V. A. Krylov, G. Moser, S. B. Serpico, and J. Zerubia, "Supervised High-Resolution Dual-Polarization SAR Image Classification by Finite Mixtures and Copulas", IEEE Journal of Selected Topics in Signal Processing, Vol. 5, No. 3, pp. 554 - 566, 2011.

[17] S. Hachicha and F. Chaabene, "SAR change detection using Rayleigh copula", Proceedings of SPIE - Remote Sensing of the Coastal Ocean, Land, and Atmosphere Environment, Vol. 7858, pp. 78581F-1, 2010.

[18] J. Kitchen and W. Moran, “Copula techniques in wireless communications”, ANZIAM Journal, Vol. 51, pp. C 526 - C540, 2010.

[19] J. A. Ritcey, "Copula Models for Wireless Fading and their Impact on Wireless Diversity Combining", the Forty-First Asilomar Conference on Signals, Systems and Computers, pp. 1564 - 1567, 2007.

[20] F. Downton, "Bivariate exponential distributions in reliability theory", Journal of the Royal Statistical Society, Series B (Methodological), Vol. 32, No. 3, pp. $408-417,1970$.

[21] J. Hunter, "Markovian queues with correlated arrival processes". Asia-Pacific Journal of Operational Research 24, pp. 593 - 611, 2007.

[22] N. C. Sagias and G. K. Karagiannidis, "Gaussian class multivariate Weibull distributions: theory and applications in fading channels", IEEE Trans. on Information Theory, pp. $3608-3619,2005$.

[23] Y. Chen and C. Tellambura, "Distributions functions of selection combiner output in equally correlated Rayleigh, Rician, and Nakagami-m fading channels", IEEE Trans. on Communications, Vol. 52, No. 11, pp. 1948 -1956, 2004. 
[24] M. S. Alouini and M. K. Simon, "Dual diversity over correlated log-normal fading channels”, IEEE Trans. on Communications, pp. 1946 - $1959,2002$.

[25] M.K. Simon and M.S Alouini, Digital Communication over Fading Channels, 2nd ed., New York: John Wiley \& Sons, 2005.

[26] M. R. Patel, Wind and Solar Power Systems, Florida, CRC Press, 1999.

[27] N. M. Temme, “A Double Integral Containing the Modified Bessel Function: Asymptotics and Computation”, Mathematics of Computation, Vol. 47, No. 176, pp. $683-691,1986$.

[28] M. Abramowitz and I. A. Stegun, Handbook of Mathematical Functions with Formulas, Graphs, and Mathematical Tables, New York: Dover, 1974.

[29] A., Nuttall, "Some integrals involving the Q_M function”, IEEE Transactions on Information Theory, Vol. 21, No. 1, pp. 95 - $96,1975$.

[30] M.K. Simon, Probability distributions involving Gaussian random variables, New York: Springer-Verlag, 2006.

[31] M.K, Simon, "Comments on "Infinite-Series representations associated with the bivariate Rician distribution and their applications", IEEE Trans. on Communications, vol. 54, no.8, pp. 1151-1152, 2006.

[32] W.H. Press, S. A. Teukolsky, W. T. Vetterling, B.P. Flannery, Numerical Recipes in C-The Art of Scientific Computing, 2nd ed., Cambridge, U.K., Cambridge Univ. , 1992.

[33] C. Tellambura and A. D. S. Jayalath, “Generation of Rayleigh and Nakagami-m fading envelopes”, IEEE Communications Letters, Vol. 4, No. 5, pp. 170 - 172, 2000.

[34] P. Dharmawansa,Member, N. Rajatheva and C. Tellambura, “On the Trivariate Rician Distribution”, IEEE Transactions on communications, Vol. 56, No. 12, pp. 1993 - 1997, 2008.

[35] W. K. Pratt, "Partial differentials of Marcum's Q function", Proceedings of the IEEE, Vol. 56, No. 7, pp. 1220 - 1221, 1968. 


\section{List of Figure Captions:}

Fig. 1: Bivariate Weibull/exponential/Rayleigh copula

Fig. 2: Bivariate Nakagami-m copula

Fig. 3: Bivariate Rician copula

Fig. 4: Bivariate Weibull/exponential/Rayleigh copula density

Fig. 5: Bivariate Nakagami-m copula density

Fig. 6: Bivariate Rician copula density 
TABLE 1: SUMMARY OF EXISTING RESEARCH AND OUR WORKS IN THIS PAPER

\begin{tabular}{ccccc}
\hline \multirow{2}{*}{ Copulas } & \multicolumn{2}{c}{ Copula function } & \multicolumn{2}{c}{ Copula Density function } \\
\cline { 2 - 5 } & Existing & Our work & Existing & Our work \\
\hline Exponential & N/A & Eq. (10) and (14) & N/A & Eq. (28) \\
\hline Rayleigh & {$[4][5]$} & Deriving new forms in Eq. (10) and (14) & N/A & Eq. (28) \\
\hline Weibull & N/A & Eq. (10) and (14) & N/A & Eq. (28) \\
\hline Log-normal & {$[5]$} & To prove log-normal copula equals to & N/A & Eq. (27) \\
\hline Nakagami-m & {$[6]$} & Gaussian copula. See Eq. (13) & & \\
\hline Rician & N/A & Using results in [6], see Eq. (19) & N/A & Eq. (30) \\
\hline
\end{tabular}

* Note that it is proven in this paper that bivariate exponential, Rayleigh and Weibull copulas are equivalent

** N/A in cells represents unexplored areas. 


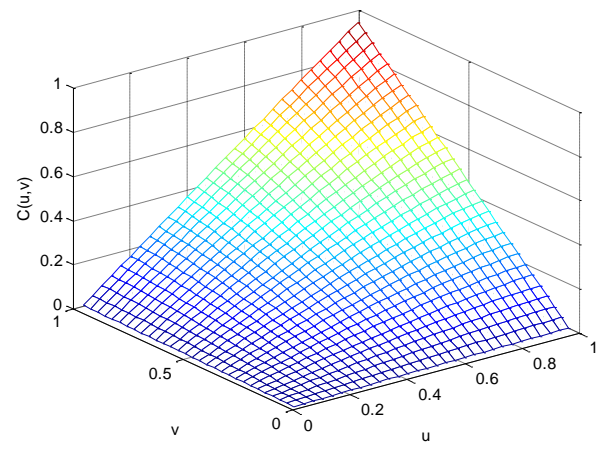

a:Bivariate Weibull/exponential/Rayleigh copula with parameter $\rho=0.3$

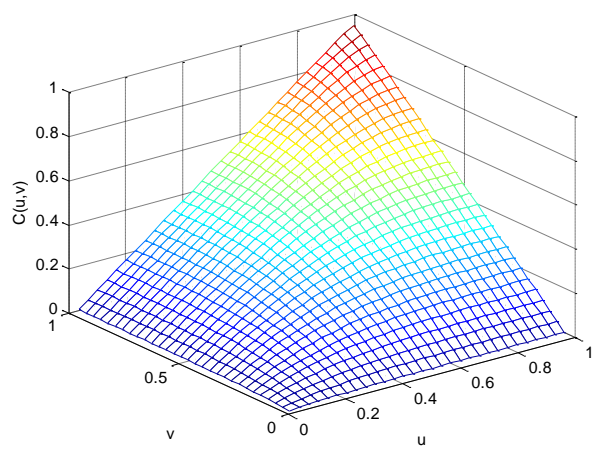

c: Bivariate Weibull/exponential/Rayleigh copula with parameter $\rho=0.8$

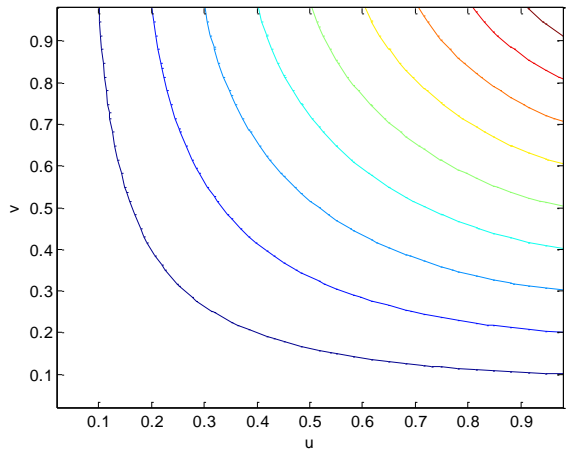

b: Contour plot of Bivariate Weibull/exponential/Rayleigh copula with parameter $\rho=0.3$

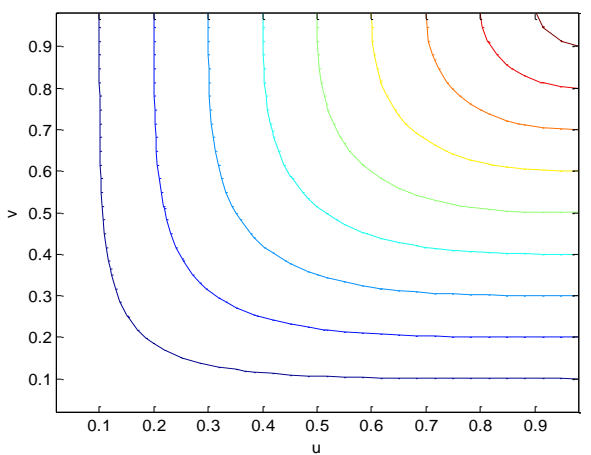

d: Contour plot of Bivariate Weibull/exponential/Rayleigh copula with parameter $\rho=0.8$

Fig. 1: Bivariate Weibull/exponential/Rayleigh Copula 


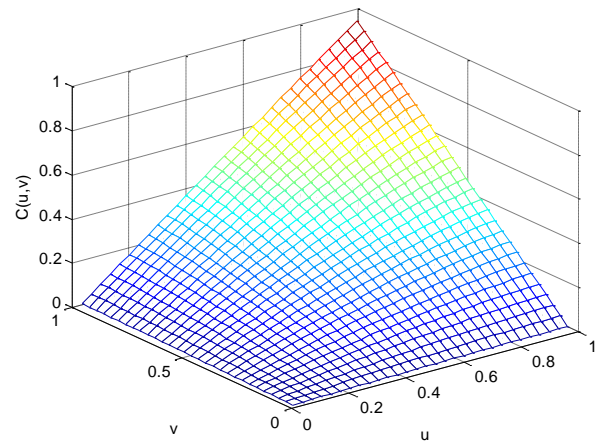

a: Bivariate Nakagami-m copula with parameter $\rho=0.3 ; m=2$

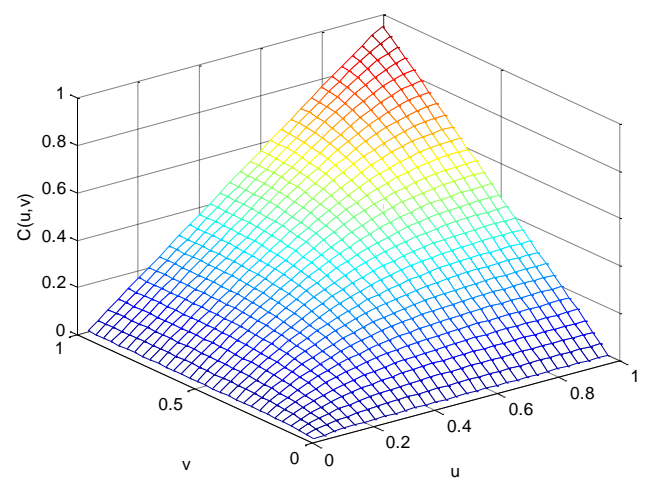

c: Bivariate Nakagami-m copula with parameter $\rho=0.8 ; m=2$

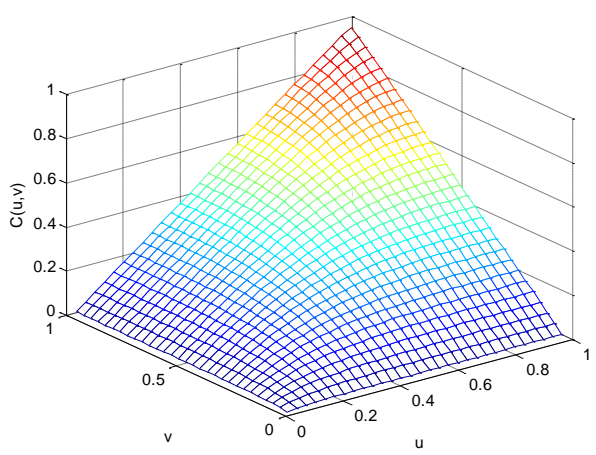

e: Bivariate Nakagami-m copula with parameter $\rho=0.8 ; m=5$

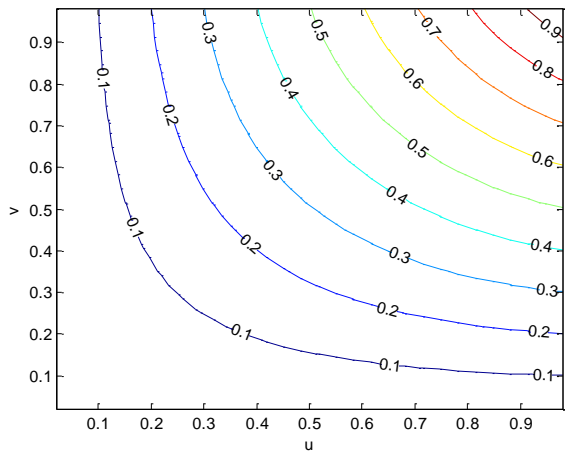

b: Contour plot of Bivariate Nakagami-m copula with parameter $\rho=0.3 ; m=2$

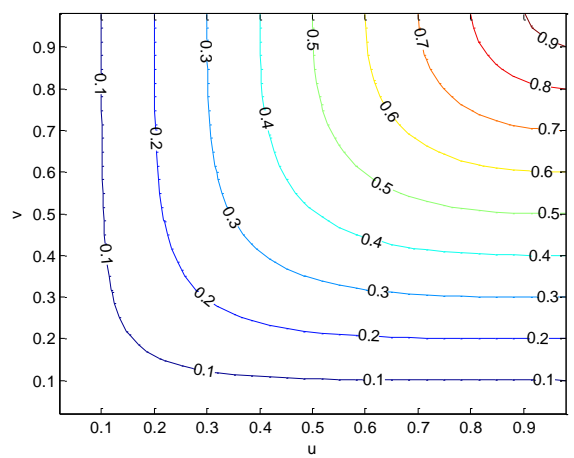

d: Contour plot of Bivariate Nakagami-m copula with parameter $\rho=0.8 ; m=2$

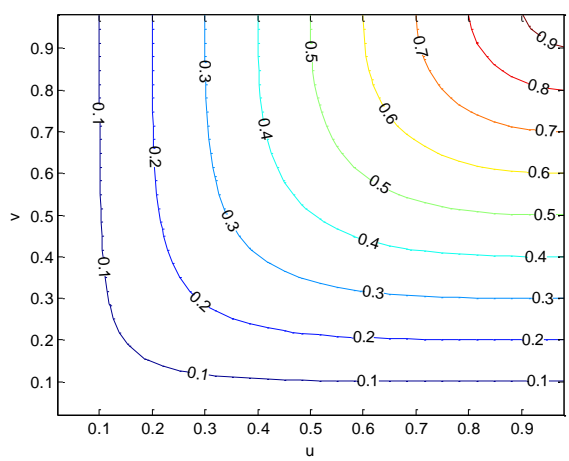

f: Contour plot of Bivariate Nakagami-m copula with parameter $\rho=0.8 ; m=5$

Fig. 2: Bivariate Nakagami-m Copula 


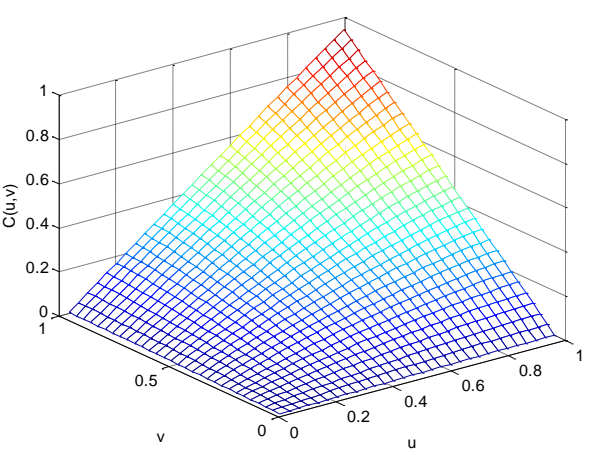

a: Bivariate Rician copula with parameter $\rho=0.3 ; \mathrm{z}=0.5$;

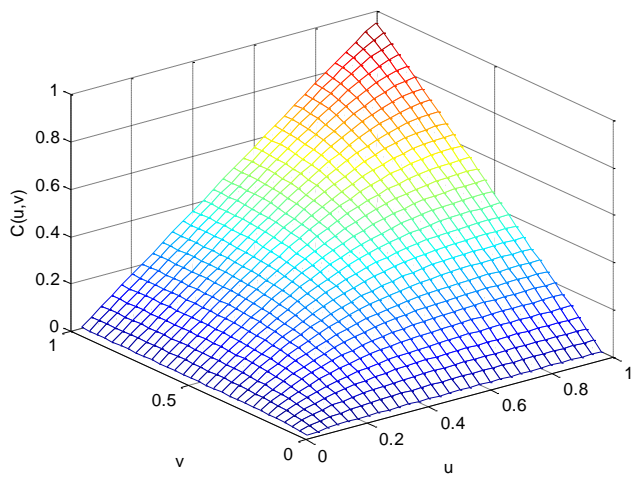

c: Bivariate Rician copula with parameter $\rho=0.3 ; z=2$

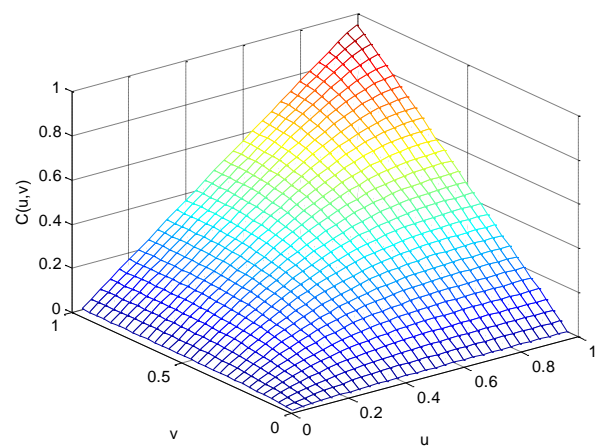

e: Bivariate Rician copula with parameter $\rho=0.8 ; \mathrm{z}=2$

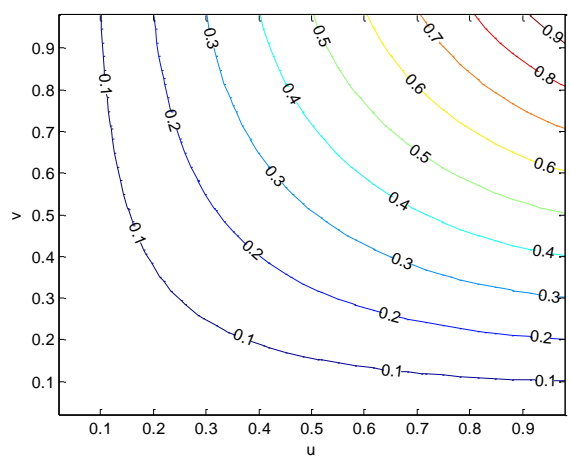

b: Contour plot of Bivariate Rician copula with parameter $\rho=0.3 ; \mathrm{z}=0.5$

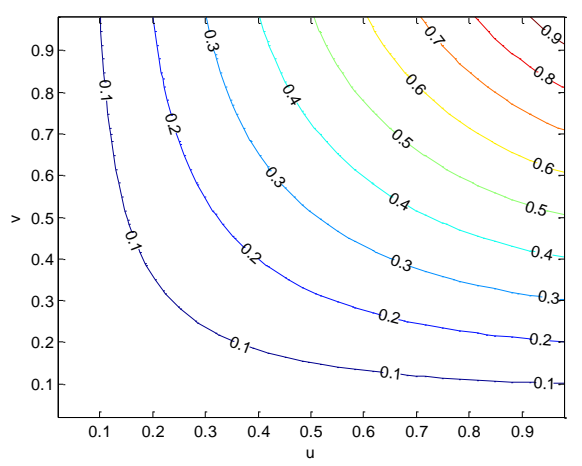

$\mathrm{d}$ : Contour plot of Bivariate Rician copula with parameter $\rho=0.3 ; z=2$

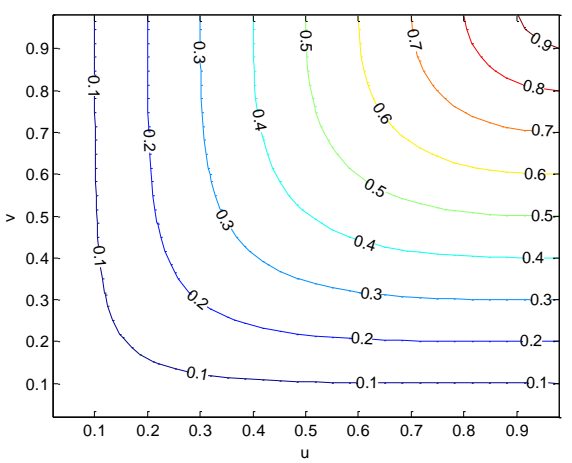

f: Contour plot of Bivariate Rician copula with parameter $\rho=0.8 ; \mathrm{z}=2$

Fig. 3: Bivariate Rician Copula 


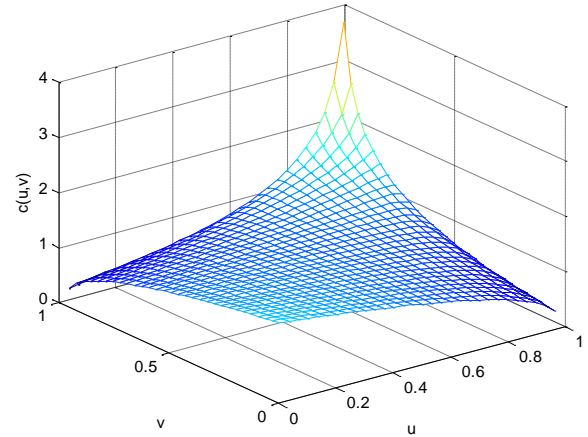

a: Bivariate Weibull/exponential/Rayleigh copula with parameter $\rho=0.3$

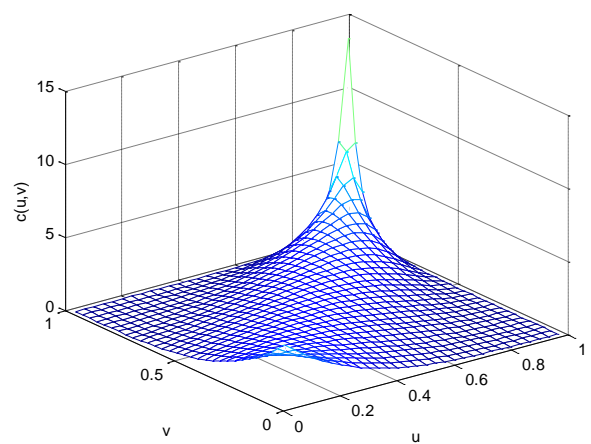

b: Bivariate Weibull/exponential/Rayleigh copula with parameter $\rho=0.8$

Fig. 4: Bivariate Weibull/expontial/Rayleigh copula density 


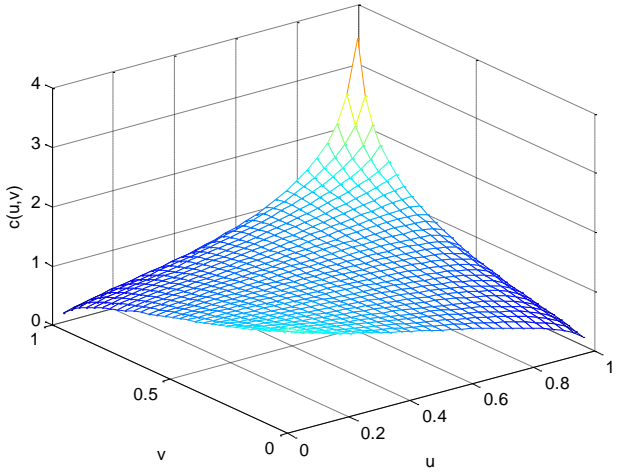

a: Bivariate Nakagamik-m copula density with parameter $\rho=0.3 ; m=2$

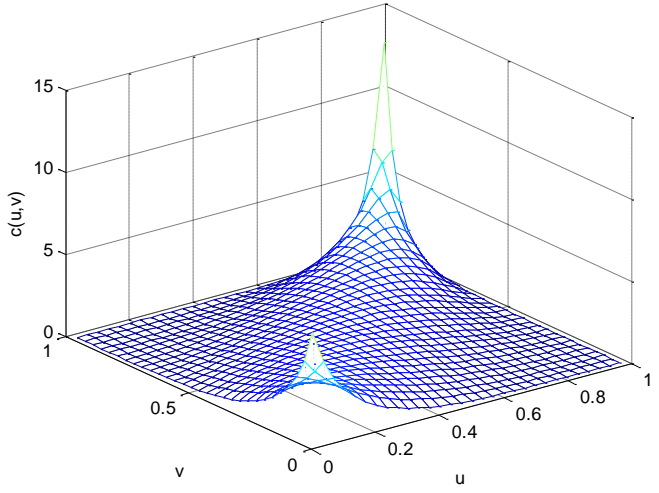

b: Bivariate Nakagamik-m copula density with parameter $\rho=0.8 ; m=2$

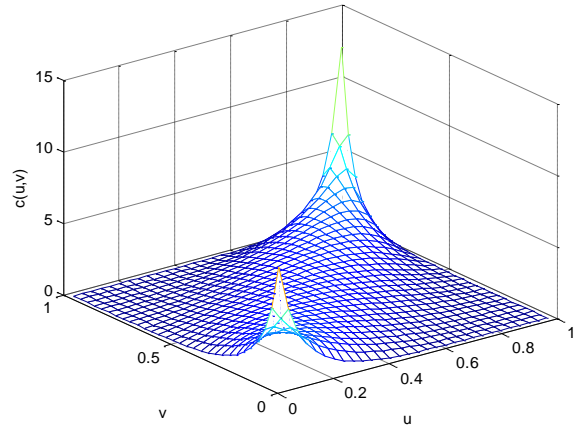

c: Bivariate Nakagamik-m copula with parameter $\rho=0.8 ; m=5$

Fig. 5: Bivariate Nakagami-m copula density 


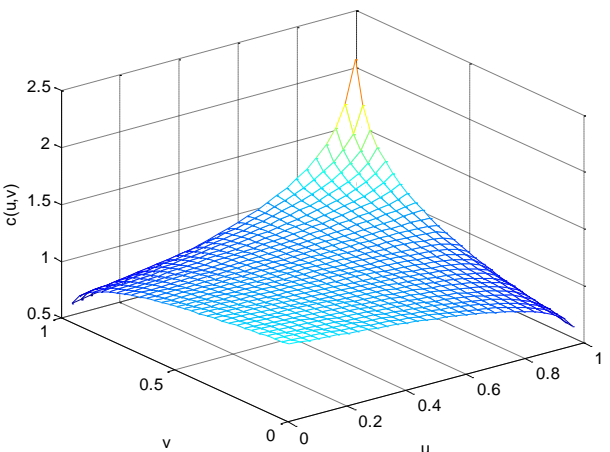

a: Bivariate Rician copula with parameter $\rho=0.3 ; z=0.5$

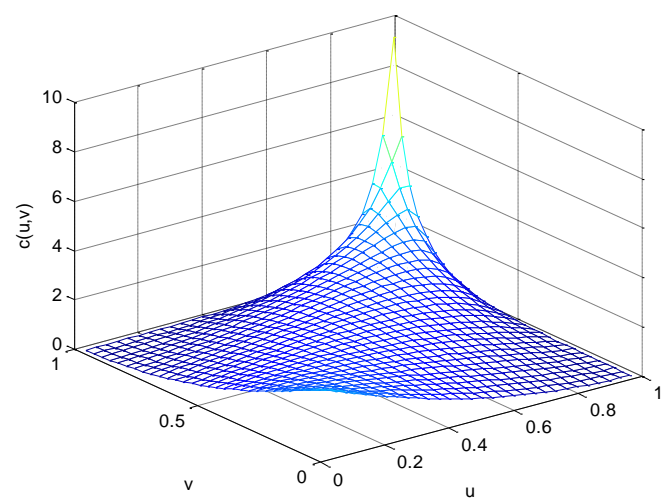

b: Bivariate Rician copula with parameter $\rho=0.8$; $z=0.5$

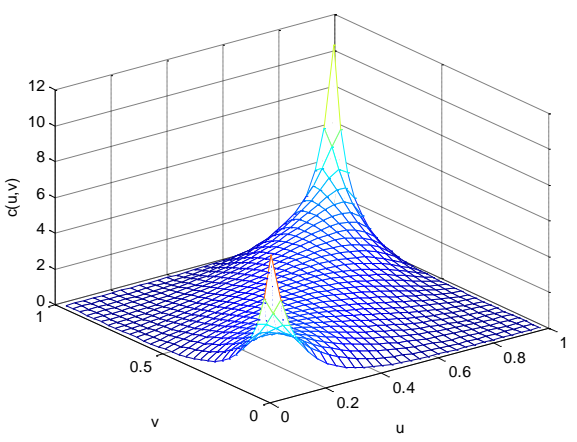

c: Bivariate Rician copula with parameter $\rho=0.8$; $z=2$

Fig. 6: Bivariate Rician copula density 